\title{
Longitudinal Association between Prostatitis and Development of Benign Prostatic Hyperplasia
}

\author{
Jennifer L. St. Sauver, PhD1 ${ }^{1}$, Debra J. Jacobson, MS², Michaela E. McGree ${ }^{2}$, Cynthia J. \\ Girman, DrPH ${ }^{3}$, Michael M. Lieber, MD ${ }^{4}$, and Steven J. Jacobsen, MD, PhD $^{5}$ \\ 1 Mayo Clinic, Division of Epidemiology, 200 First St SW, Rochester, MN, 55905 \\ 2Mayo Clinic, Division of Biostatistics, 200 First St SW, Rochester, MN, 55905 \\ 3Merck \& Co, 351 North Sumneytown Pike, North Wales, PA, 19454 \\ 4Mayo Clinic, Department of Urology, 200 First St SW, Rochester, MN, 55905 \\ 5Southern California Permanente Medical Group, Research and Evaluation, 100 S Low Robles, 2nd Floor, \\ Pasadena, CA, 91101
}

\section{Abstract}

OBJECTIVES-To determine whether physician-diagnosed prostatitis was associated with later development of development of BPH-associated events in a longitudinal, population-based sample of 2447 men residing in Olmsted County, Minnesota.

METHODS-Medical records were reviewed for physician diagnosis of prostatitis and subsequent diagnoses of $\mathrm{BPH}$, enlarged prostate, prostatism, and acute urinary retention. Records were also reviewed for medical or surgical treatments for BPH. Odds ratios were calculated to assess the associations between physician-diagnosed prostatitis and later development of development of BPHassociated events.

RESULTS-Physician-diagnosed prostatitis was associated with a 2.4-fold increased odds of receiving a later diagnosis of prostatism, enlarged prostate, or BPH (OR: 2.44, 95\% CI: 1.48, 4.01). Prostatitis was also associated with a $70 \%$ increased odds of requiring later treatment for BPH (OR: 1.69, 95\% CI: 1.28, 2.22), and a non-significant increased odds of acute urinary retention (OR: 1.33, $95 \%$ CI: 0.89, 1.99).

CONCLUSIONS-Physician-diagnosed prostatitis was associated with an increased risk of later onset of several BPH-associated events. Physician-diagnosed prostatitis may therefore be an early marker or a risk factor for development of later prostatic or urologic problems.

\section{Keywords}

benign prostatic hyperplasia; prostatitis; temporal association

\section{INTRODUCTION}

Prostatitis is the most common presenting diagnosis for men less than 50 years of age in outpatient urology clinics ${ }^{1}$, with an estimated prevalence ranging from approximately $2 \%$ to $16 \%$, depending on the population studied, and the definition used. ${ }^{2,3}$ Hallmark symptoms include pelvic pain and voiding symptoms, which significantly impact quality of life, ${ }^{4}$ and result in annual estimated costs of $\$ 84$ million. $^{5}$

Benign prostatic hyperplasia (BPH) is also a common clinical syndrome among aging men that is typically diagnosed with the onset of lower urinary tract symptoms (LUTS), increased 
prostate size, and decreased urinary flow rates in the absence of other causes 6,7 . In Olmsted County, MN, almost half of all men will develop LUTS as they age. ${ }^{8,9}$ There is not a perfect correlation between histologic BPH and development of LUTS, as some men develop histologic BPH, but not LUTS, while other men develop LUTS in the absence of significant histologic BPH. ${ }^{10}$ However, these data indicate that development of BPH-associated events is very common among aging men.

Symptoms of prostatitis and symptoms of BPH overlap, and it can be difficult to determine whether one syndrome precedes the other, whether the two syndromes may exist concomitantly, or whether prostatitis might serve as a marker or risk factor for development of BPH-associated events. A number of investigators have found that men who have received a diagnosis of prostatitis are more likely to also receive a diagnosis of BPH compared to men who have never been diagnosed with prostatitis $5,11-13$ However, these studies tended to be cross-sectional, where both prostatitis and BPH were measured at the same time, or they asked participants to recall prostatitis diagnoses that had occurred many years prior to assessing BPHassociated events.

It has therefore been difficult to determine whether prostatitis might precede the development of BPH-associated events, and, as such, may be a marker or risk factor for later urologic problems. To address this question, we examined longitudinal data available in the Olmsted County Men's Health Study to determine whether physician-diagnosed prostatitis was associated with later diagnosis of BPH-associated events.

\section{MATERIAL AND METHODS}

\section{Study Population}

Details related to the study population have been previously published. $8,14,15$ Briefly, a cohort of Caucasian men aged 40 to 79 was randomly selected from the 1990 Olmsted County, Minnesota population. After excluding men with pre-existing urologic conditions, 3874 men were asked to join the study, and $2115(55 \%)$ agreed to participate. The cohort was actively followed on a biennial basis for fourteen years.

Questionnaires were reviewed manually for completeness and consistency upon receipt. Discrepancies were resolved by the data coordinator via telephone contact. Data were entered into an electronic data base and subjected to on-line range and consistency checks.

\section{Assessment of Prostatitis}

Physician-diagnosed prostatitis was defined as the first medical record diagnosis of acute or chronic prostatitis occurring at any time prior to the onset of BPH-associated events. Details of how prostatitis was identified have been previously described. ${ }^{3}$ Briefly, all medical records of study participants who had received a diagnosis of prostatitis, or who had reported having had prostatitis on the questionnaires, were reviewed. Prostatitis diagnoses were confirmed and clinical symptoms, laboratory findings, and text associated with the diagnosis were abstracted. The most prevalent symptoms observed during these episodes included pain in the lower back or urogenital region (94\%), dysuria (40\%), and frequency (35\%). ${ }^{16}$ Concordances between data abstractors for assessing the occurrence of physician diagnosed acute and chronic prostatitis in a sub-study of 100 cases were $96 \%$ and $100 \%$, respectively.

\section{Measurement of BPH-associated events}

Medical records were reviewed for diagnoses of prostatism, enlarged prostate, and BPH that occurred any time during the participant's residence in Olmsted County, MN. Information on acute urinary retention and treatment for BPH (medications and surgery/procedures) was also 
abstracted. Concordance between data abstractors for assessing diagnoses of prostatism, enlarged prostate or BPH in a sub-sample of 100 cases was $93 \%$.

\section{Statistical Analysis}

Baseline characteristics of men with and without prostatitis were compared using MantelHaenszel chi-square tests. Logistic regression was used to estimate associations between physician-diagnosed prostatitis with BPH related-events. Multivariable models were used to adjust for age in 1990, number of baseline physician visits, and treatment for BPH.

\section{RESULTS}

$265(10.8 \%)$ participants received a physician diagnosis of prostatitis prior to any occurrence of $\mathrm{BPH}$-associated events. The median time from the diagnosis of prostatitis to a $\mathrm{BPH}$ associated event (a physician diagnosis of prostatism, enlarged prostate, or BPH) was 8.1 years (range: 0.0-48.7 years). The median time from a physician diagnosis of prostatitis to BPH treatment was 9.8 years (range: $0.0-54.0$ ), while the median time from a physician diagnosis of prostatitis to acute urinary retention was 6.0 years (range: $0.0-55.9$ ).

Men with physician-diagnosed prostatitis were more likely to later have a medical record report of prostatism, $\mathrm{BPH}$, or enlarged prostate compared to those without a physician diagnosis of prostatitis $(90 \%$ vs $78 \%, \mathrm{p}<0.0001$; Table 1$)$. These men were also more likely to later receive treatment for BPH ( $32 \%$ vs $21 \%, \mathrm{p}<0.0001)$, and to develop acute urinary retention $(12 \%$ vs $8 \%, \mathrm{p}=0.01)$.

After adjusting for age, number of baseline physician visits, and BPH treatments, men with physician-diagnosed prostatitis were still more likely to have a later medical record report of $\mathrm{BPH}$, prostatism, or enlarged prostate compared to men without a diagnosis of prostatitis (OR: 2.44; 95\% CI:1.48, 4.01; Table 2). After adjusting for age and baseline physician visits, men with physician-diagnosed prostatitis were also more likely to later receive treatment for BPH (OR: $1.69,95 \%$ CI: $1.28,2.22$ ).

\section{COMMENT}

Our results suggest that physician-diagnosed prostatitis is associated with an over two-fold increased risk of later developing BPH-associated events, and a 70\% increased risk of later requiring medications or surgery for BPH. Additionally, while not statistically significant, our results also suggest that men diagnosed with prostatitis may have a $33 \%$ increased risk of developing acute urinary retention.

While a number of other studies have demonstrated associations between prostatitis and $\mathrm{BPH}$, 5,11-13 our study had the advantage of being able to note the first physician diagnosis of prostatitis that occurred in Olmsted County, MN. Additionally, we followed men a median of 8.1 years until the first diagnosis of a BPH-associated event to establish a true temporal relationship between prostatitis and these $\mathrm{BPH}-$ associated events.

It is currently unclear why prostatitis might predispose to the development of $\mathrm{BPH}$, but prostatitis is associated with acute or chronic bacterial infection in about 5-10\% of presenting cases. ${ }^{17}$ Chronic non-bacterial prostatitis or chronic pelvic pain syndrome accounts for the remaining $90-95 \%$ of presenting cases and may or may not be associated with the presence of leukocytes in the prostatic fluid. ${ }^{17}$ Therefore, while a perfect correlation does not exist between clinical symptoms of prostatitis and measures of inflammation, prostatitis may serve as a marker for prostatic infection and/or inflammation. Additionally, both chronic and acute inflammation have been frequently noted in prostate biopsy sections and in tissue obtained 
during prostatic resection for treatment of $\mathrm{BPH},{ }^{18-22}$ and it has been suggested that $\mathrm{BPH}$ may occur due to immune dysregulation. ${ }^{23}$ Inflammation has therefore been hypothesized to lead to the development of $\mathrm{BPH}$. If physician-diagnosed prostatitis reflects the presence of prostatic inflammation, prostatitis may also represent a risk factor for the development of BPH.

However, recent work by Nickel et al indicates that prostatitis (as measured by the he National Institutes of Health Chronic Prostatitis Symptom Index; CPSI) was not strongly correlated with pathologic inflammation in biopsy samples from men enrolled in the REDUCE trial. ${ }^{24}$ CPSImeasured prostatitis and physician-diagnosed prostatitis tend to have low agreement ${ }^{25}$, and it is not clear that physician-diagnosed prostatitis is a better measure of inflammation than CPSI-diagnosed prostatitis. If this is the case, alternative measures that truly correlate with prostatic inflammation need to be developed.

Alternatively, physician-diagnosed prostatitis may simply reflect an early marker of prostate problems in men who are likely to eventually develop BPH-associated events. These men may experience early problems related to the prostate, be significantly bothered by such symptoms, and be more likely to return to their clinicians when any urologic symptoms recur. Therefore, it is possible that the association between physician-diagnosed prostatitis and BPH-associated events was observed due to increased surveillance following the diagnosis of prostatitis.

However, in our analyses, we did control for number of baseline physician visits, and our results were unchanged. We also performed additional analyses controlling for presence of diabetes, coronary heart disease, and hypertension at baseline, as these conditions would represent a surrogate marker for increased physician visits. Our results were virtually unchanged after controlling for these conditions (data not shown), suggesting that surveillance bias may be less likely to account for the observed association between physician-diagnosed prostatitis and $\mathrm{BPH}$-associated events. However, we were unable to specifically for total numbers of urologic visits, and heightened urologic surveillance may have been present in some men initially diagnosed with prostatitis.

Men with physician-diagnosed prostatitis were also more likely to receive some type of BPH treatment compared to men without a diagnosis of prostatitis. This may have occurred for two reasons. First, treatment for BPH is a surrogate marker for presence of BPH, and as we saw an association between physician-diagnosed prostatitis and later diagnosis of BPH-related events, we expected to also see an association between physician-diagnosed prostatitis and later treatment for BPH. Alternatively, prostatitis itself presents a challenge to manage clinically ${ }^{26}$ As some of the symptoms of prostatitis are similar to symptoms of BPH, some $\mathrm{BPH}$ therapies represent a reasonable treatment option for managing prostatitis. ${ }^{17}$ However, the median time between diagnosis of prostatitis and treatment for BPH was 9.8 years, suggesting that a more likely explanation may be that presence of BPH treatment represents a diagnosis of BPH, rather than use of a BPH therapy for treatment of prostatitis itself.

Finally, men with physician-diagnosed prostatitis were more likely to develop acute urinary retention in a univariate analysis; however, these results were not statistically significant after adjusting for age, number of baseline physician visits, and BPH treatment. The disappearance of this association after adjustment could be attributed to low power, or that some BPH treatments reduce the risk of acute urinary retention. While not statistically significant, these results are consistent with those reported by Roehrborn et al who found that men with inflammatory infiltrates in prostate biopsies were also more likely to develop acute urinary retention. ${ }^{27}$ It is possible that an early diagnosis of prostatitis may identify men with prostatic inflammation that may then put them at increased risk for this severe outcome.

Strengths of our study include the use of data from a longitudinal, population-based group of men. This allowed us to minimize some of the selection biases that might otherwise occur in a referral population, where the prevalence of physician-diagnosed prostatitis might be 
expected to be substantially higher than that observed in the general population. In addition, the extensive medical records and long-term follow-up available for these men allowed us to detect incident prostate and urologic events, and to accurately determine the temporal relationship between prostatitis and BPH-related events.

Limitations include the difficulty of diagnosing prostatitis, as most men with prostatitis will not have objective findings of prostatic infection or inflammation. ${ }^{17}$ The CPSI has been developed to standardize methods for identifying men with chronic prostatitis ${ }^{19}$; however, previous studies in our population have demonstrated a lack of correlation between physiciandiagnosed prostatitis and CPSI-reported prostatitis. ${ }^{25}$ Additionally, objective criteria for defining prostatitis have been lacking. ${ }^{17}$ Given these difficulties, the likelihood of at least some misclassification for prostatitis is high. For example, category IV prostatitis is asymptomatic and may only be diagnosed through prostate biopsy; these cases of prostatitis would not have come to our attention. Because of these difficulties, some of the men diagnosed with prostatitis may not truly have had this syndrome, while some men with prostatitis may not have been identified. Similar problems in diagnosing BPH also exist, and at least some of our outcomes will also have been misclassified. Interestingly, despite the likelihood of misclassification in both exposure and outcome, if the misclassification was random (both prostatitis and BPH are both over- and under-diagnosed), our results would be biased toward no association. However, if the misclassification was non-random, our results may be biased in either direction.

We also only had data available for the first diagnosis of prostatitis, and were not able to identify men with multiple episodes of prostatitis versus single episodes. If prostatitis is a true risk factor for BPH-associated events, men with multiple episodes of prostatitis may be at higher risk for adverse urologic outcomes compared to men with few or no episodes. Therefore, our results may underestimate associations between prostatitis and these events. However, we examined the association between physician-diagnosed acute and chronic cases of prostatitis and found few differences in point estimates of associations between acute versus chronic prostatitis and $\mathrm{BPH}$-associated events (data not shown), suggesting that even acute prostatitis may increase the risk of developing a BPH-associated event.

The older men in our study were less likely than the younger men to have had a diagnosis of prostatitis. Since we looked for diagnoses of prostatitis essentially over the life-span of all the men in our study, we would have expected that the older men would have been as likely as the younger men to have a prostatitis diagnosis at some point in their life. It is possible that we excluded older men who had prostatitis by excluding men who had prostate surgery prior to study recruitment in 1990. If this was the case, and physician-diagnosed prostatitis is associated with an increased likelihood of prostate surgery, our results underestimate the association between prostatitis and $\mathrm{BPH}$-associated events.

Finally, our study population consisted of only Caucasian men, and our results may not be generalizable to other populations. However, there are currently no data to suggest that the pathologic processes involved in developing a BPH-associated event differ in various populations; therefore, if physician-diagnosed prostatitis is associated with development of BPH-associated events in Caucasian men, it might also be associated with the development of $\mathrm{BPH}-\mathrm{associated} \mathrm{events} \mathrm{in} \mathrm{men} \mathrm{of} \mathrm{other} \mathrm{races} \mathrm{or} \mathrm{ethnicities.}$

\section{CONCLUSION}

Taken together, our results indicate that physician-diagnosed prostatitis is associated with later development of BPH-associated events. Physician-diagnosed prostatitis may therefore serve as an early marker for later prostate and urinary problems. Alternatively, physician-diagnosed prostatitis may reflect a true risk factor for development of these conditions. 


\section{ACKNOWLEDGEMENTS}

This project was supported by research grants from the National Institutes of Health (DK58859, AR30582, and UL1 RR024150-01) and Merck Research Laboratories.

\section{REFERENCES}

1. Collins MM, Stafford RS, O'Leary MP, Barry MJ. How common is prostatitis? A national survey of physician visits. Journal of Urology 1998;159:1224-8. [PubMed: 9507840]

2. Krieger JN, Ross SO, Riley DE. Chronic prostatitis: epidemiology and role of infection. Urology 2002;60:8-12. [PubMed: 12521579]discussion 13

3. Roberts RO, Lieber MM, Rhodes T, Girman CJ, Bostwick DG, Jacobsen SJ. Prevalence of a physicianassigned diagnosis of prostatitis: the Olmsted County Study of Urinary Symptoms and Health Status Among Men. Urology 1998;51:578-84. [PubMed: 9586610]

4. McNaughton C, Mac Donald R, Wilt T. Interventions for chronic abacterial prostatitis. Cochrane Database Syst Rev. 2001CD002080

5. Pontari MA, Joyce GF, Wise M, McNaughton-Collins M. Prostatitis. J Urol 2007;177:2050-7. [PubMed: 17509285]

6. Emberton M, Andriole GL, de la Rosette J, Djavan B, Hoefner K, Vela Navarrete R, Nordling J, Roehrborn C, Schulman C, Teillac P, et al. Benign prostatic hyperplasia: a progressive disease of aging men. Urology 2003;61:267-73. [PubMed: 12597928]

7. Wei, JT.; Calhoun, EA.; Jacobsen, SJ. Benign Prostatic Hyperplasia. In: Litwin, MS.; Saigal, CS., editors. Urologic Diseases in America. 04-5512. US Department of Health and Human Services, Public Health Service, National Institutes of Health, National Institute of Diabetes and Digestive and Kidney Diseases, US Government Publishing Office; Washington D. C.: 2004. p. 43-67.

8. Chute CG, Panser LA, Girman CJ, Oesterling JE, Guess HA, Jacobsen SJ, Lieber MM. The prevalence of prostatism: a population-based survey of urinary symptoms. J Urol 1993;150:85-9. [PubMed: 7685427]

9. Sarma AV, Wei JT, Jacobson DJ, Dunn RL, Roberts RO, Girman CJ, Lieber MM, Cooney KA, Schottenfeld D, Montie JE, et al. Comparison of lower urinary tract symptom severity and associated bother between community-dwelling black and white men: the Olmsted County Study of Urinary Symptoms and Health Status and the Flint Men's Health Study. Urology 2003;61:1086-91. [PubMed: 12809866]

10. Guess HA. Benign prostatic hyperplasia: antecedents and natural history. Epidemiol Rev 1992;14:131-53. [PubMed: 1283852]

11. Barry MJ, Link CL, McNaughton-Collins MF, McKinlay JB. Overlap of different urological symptom complexes in a racially and ethnically diverse, community-based population of men and women. BJU Int. 2007doi:10.1111/j.1464-410X.2007.07191.x

12. Collins MM, Meigs JB, Barry MJ, Walker Corkery E, Giovannucci E, Kawachi I. Prevalence and correlates of prostatitis in the health professionals follow-up study cohort. Journal of Urology 2002;167:1363-6. [PubMed: 11832733]

13. Sutcliffe S, Giovannucci E, De Marzo AM, Willett WC, Platz EA. Sexually transmitted infections, prostatitis, ejaculation frequency, and the odds of lower urinary tract symptoms. American Journal of Epidemiology 2005;162:898-906. [PubMed: 16177142]

14. Jacobsen SJ, Girman CJ, Guess HA, Panser LA, Chute CG, Oesterling JE, Lieber MM. Natural history of prostatism: factors associated with discordance between frequency and bother of urinary symptoms. Urology 1993;42:663-71. [PubMed: 7504848]

15. Oesterling JE, Jacobsen SJ, Chute CG, Guess HA, Girman CJ, Panser LA, Lieber MM. Serum prostate-specific antigen in a community-based population of healthy men. Establishment of agespecific reference ranges. JAMA 1993;270:860-4. [PubMed: 7688054]see comment

16. Roberts RO, Jacobsen SJ, Jacobson DJ, Reilly WT, Talley NJ, Lieber MM. Natural history of prostatism: high American Urological Association Symptom scores among community-dwelling men and women with urinary incontinence. Urology 1998;51:213-9. [PubMed: 9495700] 
17. Habermacher GM, Chason JT, Schaeffer AJ. Prostatitis/chronic pelvic pain syndrome. Annual Review of Medicine 2006;57:195-206.

18. Gerstenbluth RE, Seftel AD, MacLennan GT, Rao RN, Corty EW, Ferguson K, Resnick MI. Distribution of chronic prostatitis in radical prostatectomy specimens with up-regulation of bcl-2 in areas of inflammation. J Urol 2002;167:2267-70. [PubMed: 11956490]

19. Litwin MS, McNaughton-Collins M, Fowler FJ Jr. Nickel JC, Calhoun EA, Pontari MA, Alexander RB, Farrar JT, O'Leary MP, Chronic Prostatitis Collaborative Research Network. The National Institutes of Health chronic prostatitis symptom index: development and validation of a new outcome measure. J Urol 1999;162:369-75. [PubMed: 10411041]

20. Anim JT, Udo C, John B. Characterisation of inflammatory cells in benign prostatic hyperplasia. Acta Histochem 1998;100:439-49. [PubMed: 9842422]

21. Wang W, Bergh A, Damber JE. Chronic inflammation in benign prostate hyperplasia is associated with focal upregulation of cyclooxygenase-2, Bcl-2, and cell proliferation in the glandular epithelium. Prostate 2004;61:60-72. [PubMed: 15287094]

22. Di Silverio F, Gentile V, De Matteis A, Mariotti G, Giuseppe V, Luigi PA, Sciarra A. Distribution of inflammation, pre-malignant lesions, incidental carcinoma in histologically confirmed benign prostatic hyperplasia: a retrospective analysis. Eur Urol 2003;43:164-75. [PubMed: 12565775]

23. Kramer G, Mitteregger D, Marberger M. Is benign prostatic hyperplasia (BPH) an immune inflammatory disease? Eur Urol 2007;51:1202-16. [PubMed: 17182170]

24. Nickel JC, Roehrborn CG, O’Leary MP, Bostwick DG, Somerville MC, Rittmaster RS. Examination of the relationship between symptoms of prostatitis and histological inflammation: baseline data from the REDUCE chemoprevention trial. J Urol 2007;178:896-900. [PubMed: 17632164]discussion 900-1

25. Roberts RO, Jacobson DJ, Girman CJ, Rhodes T, Lieber MM, Jacobsen SJ. Low agreement between previous physician diagnosed prostatitis and national institutes of health chronic prostatitis symptom index pain measures. Journal of Urology 2004;171:279-83. [PubMed: 14665894]

26. Nickel JC. The three As of chronic prostatitis therapy: antibiotics, alpha-blockers and antiinflammatories. What is the evidence? BJU International 2004;94:1230-3. [PubMed: 15610095]see comment

27. Roehrborn C, Kaplan SA, Noble WD, Lucia MS, Slawin KM, McVary KT, Kusek JW, Nyberg LM Jr. The impact of acute or chronic inflammation in baseline biopsy on the risk of clinical progression of BPH: results of the MTOP study (abstract). J Urol 2005;173:204. [PubMed: 15592076] 
Table 1

Characteristics of men with and without physician-diagnosed prostatitis

\begin{tabular}{|l|c|c|c|}
\hline Characteristic & Prostatitis N (\%) & No Prostatitis N (\%) & p-value \\
\hline Age in 1990 & & & \\
\hline $40-49$ & $83(42.3)$ & $1060(47.1)$ & 0.79 \\
\hline $50-59$ & $56(28.6)$ & $544(24.2)$ & \\
\hline $60-69$ & $42(21.4)$ & $406(18.0)$ & \\
\hline $70+$ & $15(7.7)$ & $241(10.7)$ & \\
\hline More than one physician visit per year (at baseline) & $96(49.2)$ & $946(42.6)$ & 0.07 \\
\hline Treatments (surgery or medications for BPH) & $96(32.1)$ & $444(20.7)$ & $<0.0001$ \\
\hline $\begin{array}{l}\text { Physician diagnosis of prostatism, enlarged prostate, or benign prostatic } \\
\text { hyperplasia }\end{array}$ & $176(89.8)$ & $1745(77.5)$ & $<0.0001$ \\
\hline Acute urinary retention & $36(11.9)$ & $164(7.7)$ & 0.01 \\
\hline
\end{tabular}

* Mantel-Haenszel chi-square p-value 
Table 2

Multivariate-adjusted associations between physician-diagnosed prostatitis and BPH-associated events BPH-associated events

\begin{tabular}{|c|c|}
\hline Physician diagnosis of prostatism, enlarged prostate, or benign prostatic hyperplasia & N \\
\hline
\end{tabular}

\begin{tabular}{|r|r|}
\hline Treatment (surgery or medications) & 2447 \\
\hline
\end{tabular}

Acute urinary retention

Odds Ratio $(95 \%$ CI)

* Adjusted for age, baseline physician visits, and BPH treatment

${ }^{\dagger}$ Adjusted for age and baseline physician visits.

$2.44(1.48,4.01)$

$1.33(0.89,1.99)^{*}$ 